\title{
Characterization of the human zona pellucida from fertilized and unfertilized eggs
}

\author{
R. B. Shabanowitz and M. G. O'Rand* \\ Department of Obstetrics \& Gynecology, and * Laboratories for Cell Biology, \\ Department of Anatomy, University of North Carolina, Chapel Hill, NC 27514, U.S.A.
}

\begin{abstract}
Summary. Zonae pellucidae were isolated from a variety of human eggs collected from follicular aspirates for in-vitro fertilization. Zonae were removed from pools of eggs classified as fertilized but unsuitable for embryo transfer, inseminated but not fertilized, and immature and not inseminated. Isolated zonae were heat solubilized, iodinated and separated by two-dimensional electrophoresis. Under reducing conditions, zonae from unfertilized eggs separated into three acidic proteins with molecular weight ranges of $90000-110000$ (ZP1), $64000-78000$ (ZP2) and $57000-73000$ (ZP3). Under nonreducing conditions, $Z P 1$ and $Z P 2$ co-migrated at $M_{\mathrm{r}} 92000-120000$. An identical pattern was seen from zonae isolated from eggs that were not inseminated. Therefore, if chemical modification of the zona is effected by spermatozoa, these changes were not apparent in the electrophoretic patterns. The electrophoretic pattern of zonae isolated from eggs classified as fertilized revealed fertilization-associated modification of the zona pellucida. This was expressed as a modification of the ZP1 molecule, and was only evident after reduction of the sample. We suggest that this modification may be effected by egg cortical granule dehiscence after fertilization and that the chemical modification of the zona may be involved in a zona block to polyspermy.
\end{abstract}

Keywords: Zona pellucida; fertilization; human; electrophoresis

\section{Introduction}

The mammalian zona pellucida is a unique extracellular matrix which surrounds the mature oocyte (for reviews see Dunbar, 1983; Dunbar \& Wolgemuth, 1984). Once formed, the zona pellucida (ZP) remains structurally intact during ovulation, fertilization and early preimplantation embryonic development. The ZP therefore serves both as a barrier for the freshly ovulated ovum, and after fertilization, as a protective structure for the developing embryo.

Fertilization of the ovum requires attachment to, and penetration of, the ZP by the spermatozoon (for reviews see Yanagimachi, 1977; Hartmann, 1983; O'Rand et al., 1986a). In some species, the $\mathrm{ZP}$ has been shown to induce the acrosome reaction of spermatozoa, an absolute prerequisite for fertilization (Florman \& Storey, 1982; Bleil \& Wassarman, 1983; Crozet \& Dumont, 1984; Cherr et al., 1986; O'Rand \& Fisher, 1987). It has also been demonstrated that specific biochemical changes in the ZP, effected by the cortical reaction of the egg after fertilization, are responsible for the establishment of a ZP 'slow block' to polyspermy (for review see Schmell et al., 1983). Therefore, it is apparent that this non-cellular vestment of the mammalian ovum plays an important role in the regulation of sperm-egg interactions.

Although the glycoprotein components of the zona pellucida from several mammalian species have been well characterized (Bleil \& Wassarman, 1980a; Dunbar et al., 1980, 1981) there is a paucity of information characterizing the human zona pellucida. This has, in part, been due to difficulties in obtaining suitable quantities of material. With the advent of active programmes for human in-vitro fertilization and embryo transfer, however, sufficient numbers of human zonae 
pellucidae (from unfertilized eggs, or embryos classified as unsuitable for transfer) can be collected over a relatively short period of time. Additionally, it is now possible to iodinate a single zona pellucida to high specific activity and then visualize it after electrophoresis (Hedrick et al., 1987).

\section{Materials and Methods}

Isolation and solubilization of human zonae pellucidae. Human zonae pellucidae were isolated from eggs collected from follicular aspirates from patients participating in the in-vitro fertilization-embryo transfer programme at Memorial Hospital, University of North Carolina, Chapel Hill. The ZP were collected from immature eggs that were not inseminated, inseminated eggs that failed to fertilize, fertilized but fragmented eggs, polyspermic eggs, and thawedfrozen embryos classified as unsuitable for embryo transfer. These specimens were used with the patients' consent, and represent materials which would normally have been discarded.

Zona-intact eggs were rinsed and stored frozen at $-20^{\circ} \mathrm{C}$ in Dulbecco's phosphate-buffered saline (Gibco, Grand Island, N.Y.) $\mathrm{pH} 7.4$ (PBS) until use. For some experiments, zonae were prepared from fresh samples, without freezing. Fresh or thawed eggs were transferred to a washing solution of $0.5 \mathrm{M}-\mathrm{NaCl}, 0.05 \%$ Triton X-100 to remove any adherent spermatozoa or corona radiata cells, and the zonae were mechanically removed from eggs using a 26-gauge needle to break the zona and allow the cellular material to exude. This manipulation was performed while observing the specimen under a dissecting stereomicroscope at a magnification of $\times 63$. Isolated ZP were pooled and then washed once in the washing solution and twice in PBS, centrifuging at $11600 \mathrm{~g}$ for $10 \mathrm{~min}$ between washes. To the final pellet, consisting of 2-25 ZP depending upon the experiment, $100 \mu$ listilled water adjusted to $\mathrm{pH} 10 \cdot 0$ with solid $\mathrm{Na}_{2} \mathrm{CO}_{3}$ were added, and the zonae were heat solubilized for $90 \mathrm{~min}$ at $70^{\circ} \mathrm{C}$

Iodination of heat-solubilized ZP. Heat-solubilized ZP were iodinated with carrier free $\mathrm{Na}^{125} \mathrm{I}$ (ICN, Irvine, CA) using Iodobeads (Pierce Chemical Co., Rockford, IL). The iodination mixture consisted of $100 \mu 1$ solubilized ZP, $90 \mu 1$ PBS, $10 \mu \mathrm{l} \mathrm{Na}{ }^{125} \mathrm{I}(0.5 \mathrm{mCi})$, and one Iodobead. Iodination was performed in a $2-\mathrm{ml}$ microtube for $15 \mathrm{~min}$ at room temperature with gentle agitation. Labelled protein was separated from free iodide on a $15 \cdot 0-\mathrm{ml}$ bed volume of BioGel P-2 (Bio-Rad, Rockville Centre, NY), using distilled water as eluant. Fractions of $350 \mu \mathrm{l}$ were collected and $3 \mu \mathrm{l}$ aliquants assayed for radioactivity by liquid scintillation counting. Those fractions containing radiolabelled protein were pooled; pooled samples typically contained $1000-4000$ c.p.m. $/ \mu$ l.

Polyacrylamide gel electrophoresis. Isoelectric focussing (IEF) was performed according to O'Farrell (1975) with minor modifications. IEF tube gels were cast in Natelson blood collecting tubes (Fisher Scientific, Raleigh, N.C.) to a height of $8 \mathrm{~cm}$. The gels contained a total of $3 \%$ ampholytes in various ratios of $\mathrm{pH} 3-10$ and $\mathrm{pH} 3-7$ to optimize the resultant $\mathrm{pH}$ gradient for visualization of $\mathrm{ZP}$. Labelled $\mathrm{ZP}$ were mixed in O'Farrell's lysis buffer with (reducing) or without (non-reducing) 2-mercaptoethanol. The ZP samples, containing 2000-50 000 c.p.m., depending on the experiment, were added to each tube along with an aliquant of molecular weight markers (Pharmacia, Piscataway, NJ). Samples were electrofocussed at $350 \mathrm{~V}$ for $15-17 \mathrm{~h}$ followed by $700 \mathrm{~V}$ for $5 \mathrm{~min}(5300-6000$ Volt-hours), and the gels were extruded and frozen at $-70^{\circ} \mathrm{C}$ until use, usually within $24 \mathrm{~h}$. Thawed gels were equilibrated for $5 \mathrm{~min}$ in O'Farrell's equilibration buffer with or without 2-mercaptoethanol. For each individual sample analysed, two IEF gels were run under non-reducing conditions and one under reducing conditions. Before running the second dimension, one of the non-reduced IEF gels was equilibrated with 2-mercaptoethanol included in the buffer. Each sample was thereby analysed by three gels, one under non-reducing conditions in both dimensions, one under non-reducing conditions in the first dimension only, and one under reducing conditions in both dimensions.

The second dimension was run on $8.5 \mathrm{~cm} \times 8.0 \mathrm{~cm} \times 0.15 \mathrm{~cm}$ SDS-slab gels of uniform $10 \%$ or $12 \%$ acrylamide without a stacking gel. Equilibrated IEF gels were placed directly on top of the slab gel and electrophoresed at $20-25 \mathrm{~mA} / \mathrm{gel}$ constant current until a bromophenol blue marker reached the bottom of the gel. Gels were fixed and stained for $20 \mathrm{~min}$ in $50 \%$ ethanol, $10 \%$ acetic acid, $0 \cdot 2 \%$ Coomassie Blue R-250 and destained in $10 \%$ acetic acid, $5 \%$ ethanol. After destaining, gels were soaked in $1 \%$ glycerol for $30 \mathrm{~min}$ and then dried on filter paper in a gel dryer.

Dried gels were exposed to Kodak X-Omat AR film (Eastman Kodak Co., Rochester, NY) with the use of an intensifying screen. India ink, containing ${ }^{125} \mathrm{I}$, was used to mark the filter paper with several spots to allow for proper orientation of the gels with the resultant autoradiogram. Gels were exposed at $-70^{\circ} \mathrm{C}$ for $24-96 \mathrm{~h}$, depending upon the initial counts in the sample. Some gels were exposed several times for different lengths of time to visualize specific areas of the gel containing weakly radiolabelled species. Autoradiographs were developed in Kodak GBX developer and fixed in Kodak GBX fixer according to the manufacturer's directions. The internal Coomassie blue-stained molecular weight standards were used to establish a co-ordinate system for determination of both molecular weight and $\mathrm{pl}$ of unknowns.

Electroelution of zona proteins. To prepare purified fractions of ZP proteins, non-reduced samples were electrophoresed in preparative slab gels, followed by electroelution of the proteins from those specific regions of the gel containing the separated ZP components. Labelled ZP in distilled water were mixed (1:1, v/v) with O'Farrells equilibration buffer without 2-mercaptoethanol, and electrophoresed on a $14 \mathrm{~cm} \times 16 \mathrm{~cm} \times 0.15 \mathrm{~cm} 10 \%$ SDS-slab gel in the same buffer system as used for the second dimension of IEF/SDS-PAGE. After electrophoresis, the gel was carefully removed, placed on a clean glass plate, covered with plastic film and exposed to X-ray film overnight at $4^{\circ} \mathrm{C}$. 
The resultant autoradiograph was used to locate radiolabelled bands within the gel, and individual bands were cut from the gel. These gel slices, containing the radiolabelled proteins, were then subjected to electroelution using the same buffer system as described above. Eluted proteins were exhaustively dialysed against distilled water. Recovered material contained $200-1000$ c.p.m. $/ \mu$ l.

\section{Results}

\section{Electrophoretic characterization of $Z P$}

Initially, pooled ZP from a variety of eggs were characterized by 2-dimensional IEF/SDS-PAGE. Under non-reducing conditions 2 bands were visible, one with a range of $M_{\mathrm{r}} 92000-120000, \mathrm{pI}$ 4.4-4.5 and the other with a range of $M_{\mathrm{r}} 57000-73000$, pI 4.2-4.4 (Fig. 1a). Under reducing conditions, however, 3 bands became visible (Figs 1b, c), and it appeared that the higher molecular weight component seen under non-reducing conditions was actually composed of two individual species that co-migrated to the same position in the gel, and only became separated after reduction. Using the labelling system of Sacco et al. (1981) for the designation of individual ZP proteins, we refer to the three $\mathrm{ZP}$ components seen under reducing conditions as ZP1, ZP2 and ZP3 for the highest to lowest molecular weight species respectively.

After reduction with 2-mercaptoethanol and the consequent separation of the ZP1/ZP2 complex seen under non-reducing conditions, ZP1 appeared to migrate to a slightly lower molecular weight range of 90000-110000 (compare Fig. la with Fig. 1b). The relative position of ZP2 within the gel was dependent upon whether or not reduction occurred before or after IEF. When the sample was reduced after IEF, ZP2 ran directly below ZP1, to a molecular weight range of $64000-78000$, pI 4.4-4.5 (Fig. 1b). If, however, the sample was reduced before IEF, ZP2 migrated to the same molecular weight range, but a slightly higher $\mathrm{pI}$ range of $4 \cdot 5-4 \cdot 6$ (Fig. 1c).

The relative intensities of the individual $\mathrm{ZP}$ families within the autoradiographs also depended upon whether the samples were reduced. Under non-reducing conditions the ZP1/ZP2 complex was much darker than ZP3, which is expected since the complex consists of two radiolabelled co-migrating components rather than the single component of ZP3. Under reducing conditions, however, when the ZP1/ZP2 complex separated into individual ZP1 and ZP2 components, all three ZP families displayed approximately equal intensities. Each of the individual ZP families also displayed microheterogeneity; each family consisted of several charge isomers which were distinctly visible after short exposure times. ZP3 consisted of three darker bands towards the basic end of its pI range, with two lighter bands towards the acidic end. Both ZP1 and ZP2 consisted of two darker central bands flanked on either side by 1-2 lighter bands. Although the family of spots for ZP1 and ZP2 ran nearly horizontally within the gel, ZP3 ran with a slight upwards slant towards the acidic end of the gel. These multiple charge isomers are characteristic of glycoproteins, and the particular upwards slant of ZP3 is typical for glycoproteins containing sialic acid or other charged sugar moieties (Anderson \& Anderson 1979; Hsu \& Kingsbury, 1982; Pearson \& Anderson, 1983).

\section{Identification of contaminants}

To verify that the observed zona autoradiographic patterns were not due to contaminating proteins, several control studies were carried out. In this study, zonae were isolated from eggs initially used for in-vitro fertilization and were therefore incubated in culture medium containing up to $15 \%$ maternal serum. Unless the isolated zonae were sedulously soaked and washed in PBS to allow complete removal of permeant serum, they retained traces of serum proteins which were then iodinated. Therefore, occasionally, an additional band was present at $67000, \mathrm{pI} 4 \cdot 7$, and this has been identified as human serum albumin (Fig. 1a, HSA). HSA, the major protein in serum, was demonstrated by its co-migration with pure HSA in a 2-dimensional IEF/SDS-PAGE gel as well as its positive reaction with rabbit anti-human albumin (Sigma, St Louis, MO) (data not shown). 

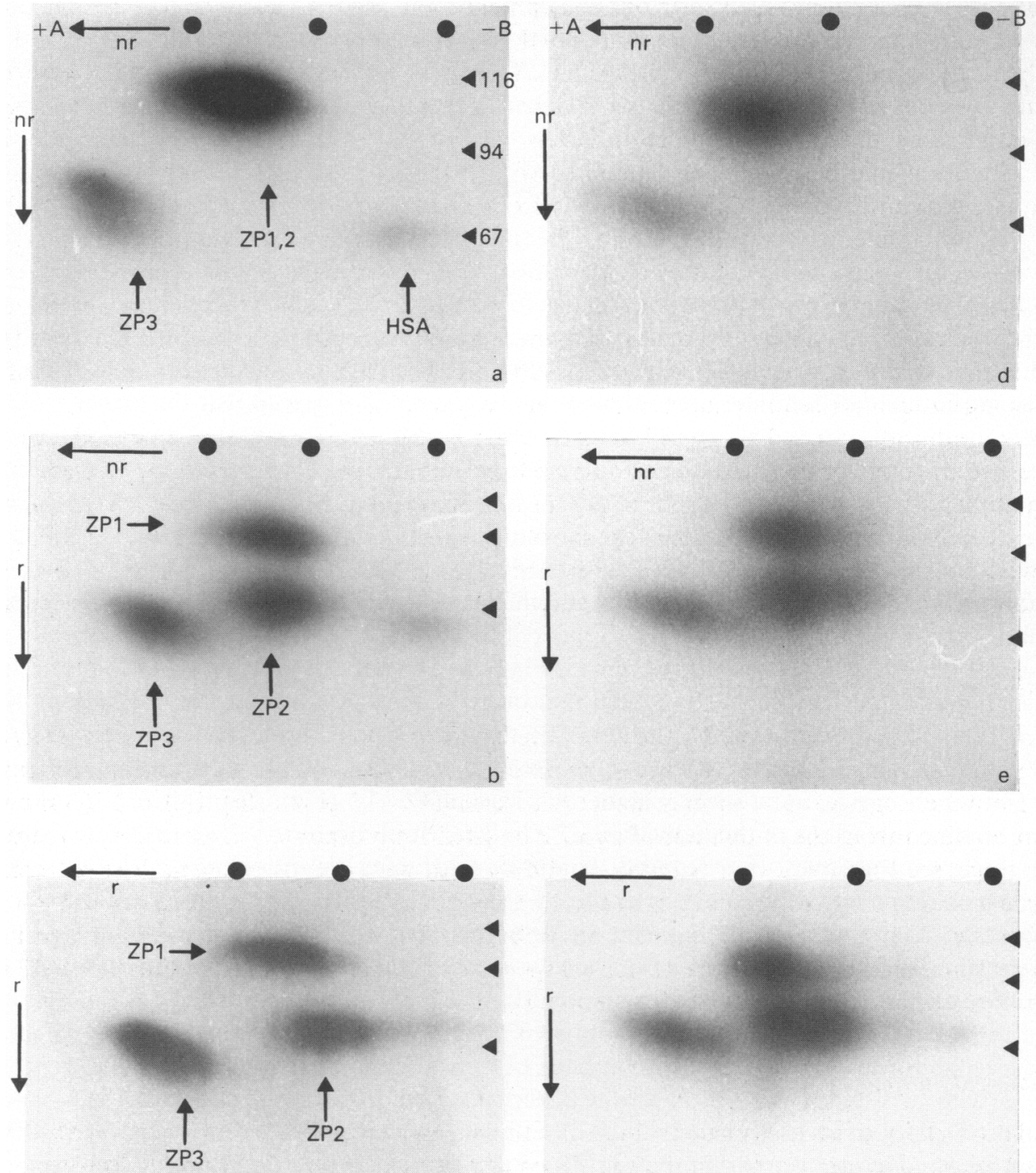

Fig. 1. Autoradiographs of ${ }^{125}$ I-labelled heat-solubilized human zonae pellucidae. Sample origin was in the upper right-hand corner of each gel. Samples were separated by 2-dimensionalIEF/SDS-PAGE. Direction of isoelectric focussing is indicated by the horizontal arrows, from the basic buffer $(-\mathbf{B})$ towards the acidic buffer $(+\mathbf{A})$. Direction of SDS-PAGE is indicated by the vertical arrows. Labelling along arrows indicates whether the sample was non-reduced (nr) or reduced ( $r$ ) in that dimension. Autoradiographs are labelled to indicate the position of markers used for $\mathrm{pI}$ and molecular weight determination. Dots along the top margin of each gel represent, from left to right, the final position of the predominant charge isomer of soybean trypsin inhibitor (pI 4.5), ovalbumin (pI 4.6) and bovine serum albumin (BSA) (pI 4.7). Arrowheads along the right margin of each gel represent positions of molecular weight markers: $\beta$-galactosidase (116000), phosphorylase b $(94000)$, BSA (67 000), and ovalbumin (43000). (a) Zonae isolated from 20 eggs that were inseminated but not fertilized. (b) Same sample as in (a) but the non-reduced IEF gel was equilibrated with 2-mercaptoethanol before running the second dimension. (c) Same sample as in (a) but the sample was reduced before 
The occasional presence of HSA in our zona preparations made us question whether the pooled zonae we were characterizing were contaminated with other non-zona materials that would mistakenly be identified as actual zona components. To resolve this potential problem of whether our washing protocol was efficiently removing attached spermatozoa, corona radiata cells, or residual egg contaminants, the entire egg, with intact zona, was iodinated, rather than isolated zonae alone. These samples would be heavily contaminated with materials that might easily be included with inadequately cleaned zonae. Autoradiographs from these samples revealed essentially the same pattern as for isolated zonae except for very heavy contamination with HSA; occasionally some lower molecular weight components were also detected (data not shown). Therefore, even when the entire egg-zona complex was iodinated, apart from HSA, there was little other contaminating material. Although one might expect to see a variety of radiolabelled proteins derived from the egg or spermatozoa in these samples, the washing buffers and centrifugation speeds between washings were strong enough for cellular material to be removed with the supernatants.

As an additional control to identify contamination with non-zona material, we iodinated the culture medium used for in-vitro culture. This medium is a modified Ham's F-10 supplemented with $15 \%$ maternal serum. Autoradiographs of this medium consisted almost entirely of HSA, which was so heavily radiolabelled that even with very short exposure times it obscured nearly everything else. However, nothing migrated to the area where the three ZP proteins would normally be present, precluding the possibility of erroneously identifying materials in the medium for zona components.

\section{Sperm-associated alterations}

Finally, a control was needed to resolve whether or not the zona itself would be altered by insemination and incubation of eggs with spermatozoa, and we used eggs that were uninseminated; zonae from these eggs were never in contact with spermatozoa. Figures 1(d), 1(e) and 1(f) show the pattern obtained from the iodination of two ZP isolated from aspirated immature eggs (germinal vesicle stage; see Figs $1 \& 2$ in O'Rand et al., 1986b) that were not inseminated. The patterns are nearly identical to those of Figs $1(\mathrm{a}), 1(\mathrm{~b})$ and $1(\mathrm{c})$, indicating that insemination alone did not alter the $\mathrm{ZP}$ in any manner detectable by the methods we used to characterize the zonae. Other experiments, involving short-term co-incubations of washed spermatozoa with solubilized radiolabelled ZP have yielded similar results (unpublished observations).

\section{Isolation and purification of labelled $Z P$}

Isolation and purification of individual ZP families was accomplished by preparative electrophoresis followed by electrophoretic elution. Two bands were detected after slab gel electrophoresis, one heavily radiolabelled band and a lighter band (ZP1/ZP2 and ZP3 respectively, Fig. 2a). This autoradiograph was used to locate the individual bands within the gel, and the bands were excised and subjected to electroelution.

The eluted samples were analysed by 2-dimensional IEF/SDS-PAGE under reducing and nonreducing conditions, and consisted of a purified fraction of $\mathrm{ZP} 3$ (Fig. 2b) and purified $\mathrm{ZP} 1$ and ZP2, which comigrated under non-reducing conditions (Fig. 2c) and became separate from one another when reduced (Fig. 2d). A band consisting of all the radiolabelled material of the preparative gel above ZP1/ZP2 was also electroeluted. This material appears to be an aggregate of all the zona families, some of which separate from the aggregate when the sample is reduced and electrophoresed

2-dimensional IEF/SDS-PAGE. (d) Zonae isolated from two eggs that were classified as immature and were not inseminated. (e) Same sample as in (d) but the non-reduced IEF gel was equilibrated with 2-mercaptoethanol before running the second dimension. (f) Same sample as in (d) but the sample was reduced before 2-dimensional IEF/SDS-PAGE. 


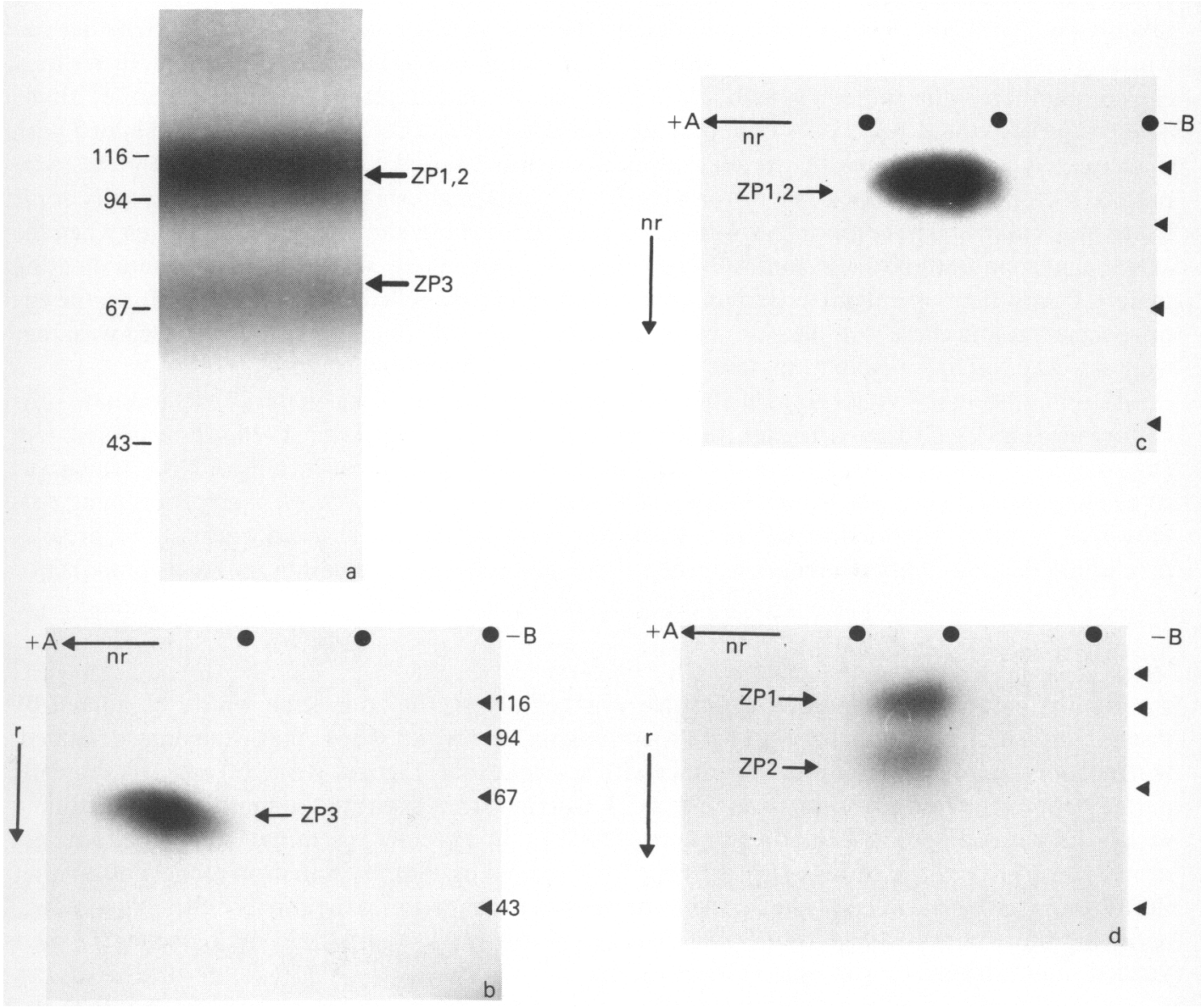

Fig. 2. Purification and isolation of individual zona proteins. ${ }^{125} \mathrm{I}$-labelled heat-solubilized zonae were electrophoresed on a preparative polyacrylamide slab gel under non-reducing conditions. Zonae families ZP1, 2 and ZP3 were cut from the preparative gel, electroeluted, and analysed by 2-dimensional IEF/SDS-PAGE. (a) Portion of autoradiograph of zona material separated under non-reducing conditions in preparative slab gel. Bands indicated as ZP1/ZP2 and ZP3 were cut from the gel and electroeluted. (b) Autoradiograph of zona material electroeluted from the band designated as ZP3 in 2(a). (c) Autoradiograph of zona material electroeluted from the band designated in ZP1/ZP2 in 2(a). (d) Same sample as in 2(c) but the non-reduced IEF gel was equilibrated with 2-mercaptoethanol before running the second dimension.

(data not shown). In other experiments, this aggregate was always present after short ( $<1 \mathrm{~h}$ ) heat solubilization times, and is significantly reduced when longer solubilization times were used (data not shown).

\section{ZP from 'fertilized', activated eggs versus 'unfertilized' eggs}

To examine the possible effects of fertilization upon the molecular structure of the ZP, zonae were segregated into two groups. One group, designated 'unfertilized', consisted of ZP isolated from inseminated eggs that did not fertilize. Specifically, these were eggs in which, at $40 \mathrm{~h}$ after 

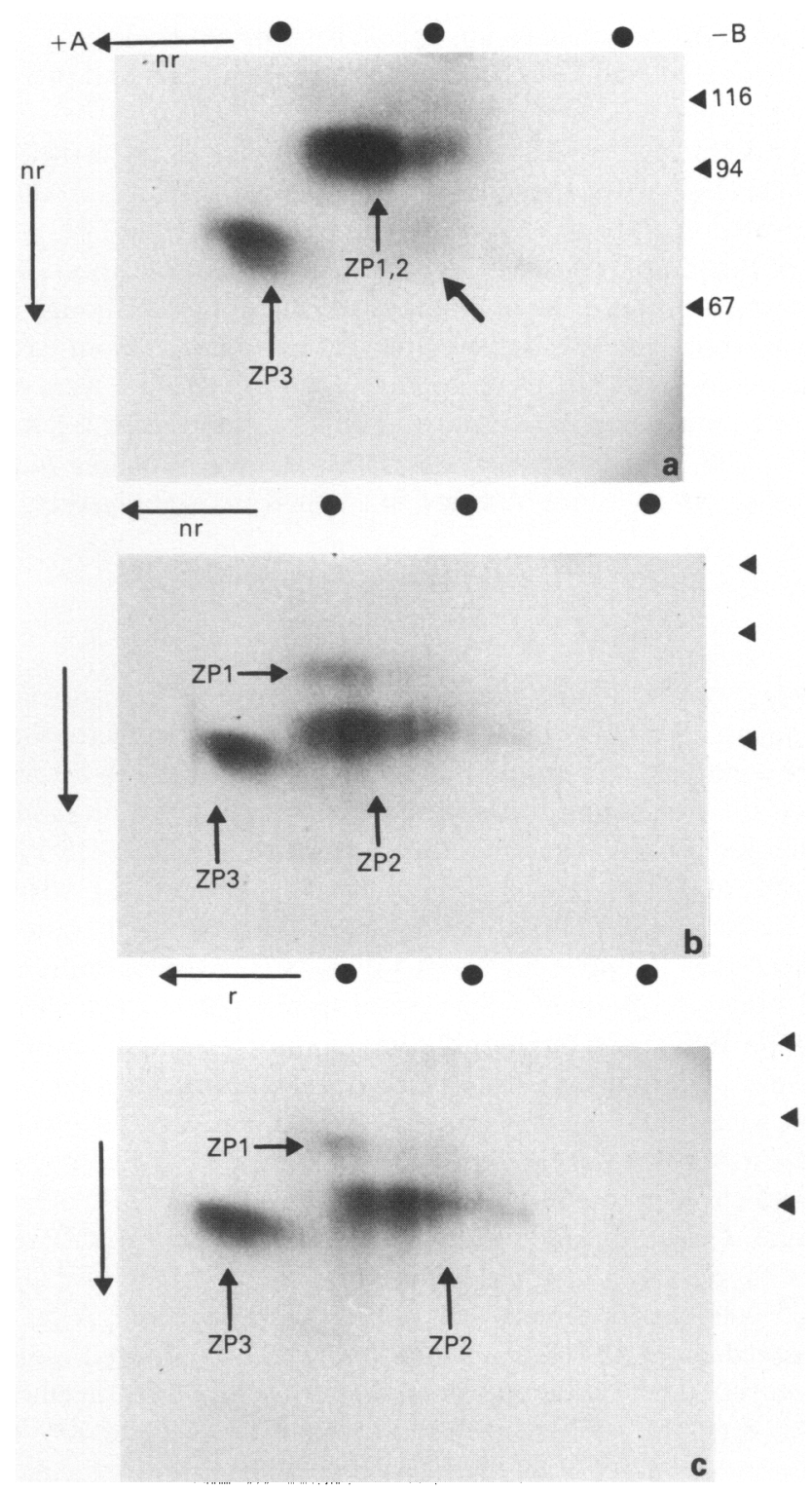

Fig. 3. Autoradiograph of ${ }^{125} \mathrm{I}$-labelled heat-solubilized zonae isolated from a pool of 13 eggs classified as fertilized. These eggs included polyspermic eggs, and eggs that were fertilized but fragmented. (a) Non-reducing conditions used in both directions of 2-dimensional IEF/SDSPAGE. Arrow points to labelled component migrating to position where reduced ZP2 typically migrates. This band was of variable intensity, and was usually very lightly labelled. (b) Same sample as in 3(a) but the non-reduced IEF gel was equilibrated with 2-mercaptoethanol before running the second dimension. (c) Same sample as in 3(a) but the sample was reduced before 2-dimensional IEF/SDS-PAGE. 
insemination a polar body was absent, or only one polar body was present. The other group, designated as 'activated' eggs, consisted of ZP isolated from inseminated eggs that became polyspermic, or embryos that had cleaved but fragmented. Additionally, some experiments included ZP from thawed-frozen embryos classified as unsuitable for embryo transfer. It was assumed that all the eggs classified as 'activated' had been penetrated by spermatozoa and therefore had undergone a cortical reaction.

All ZP from unfertilized eggs gave autoradiographs similar to those of uninseminated zonae. All contained ZP families 1-3, at approximately equal intensities. However, the autoradiographs of $\mathrm{ZP}$ isolated from activated eggs displayed a dramatic modification of ZP1, which became evident only after reduction of the sample (Fig. 3). This characteristic modification of ZP1 after reduction was consistently observed for three different pools of activated eggs. Although the degree of the loss of ZP1 did vary, this modification was a distinctive feature of zonae from fertilized eggs only. No fertilization-associated changes in the electrophoretic pattern of ZP3 were apparent, but a new component was observed migrating to a position similar to reduced $Z P 2$, but under non-reducing conditions (arrow, Fig. 3a). This component displayed variable labelling intensity, mostly being very slightly radiolabelled, and was not observed in zonae from eggs classified as unfertilized.

\section{Discussion}

The studies reported here have revealed, for the first time, fertilization-associated modifications in the structure of the human zona pellucida. Additionally, the specific electrophoretic characteristics of the structure of the zona pellucida from non-fertilized eggs have been described.

We have identified three acidic glycoproteins of the human $\mathrm{ZP}$, using iodination and electrophoresis. When solubilized ZP are reduced before 2-dimensional IEF/SDS-PAGE, the three zona components, ZP1, ZP2 and ZP3 have approximate $M_{\mathrm{r}} / \mathrm{pI}$ values of 90000-110000/4.4-4.5, $64000-78000 / 4 \cdot 5-4 \cdot 6$, and $57000-73000 / 4 \cdot 2-4 \cdot 4$. These molecular weight ranges are consistent with those reported by Sacco et al. (1981), and the $\mathrm{pI}$ values are consistent with reported pI values for iodinated and native zona glycoproteins from several species (Bleil \& Wassarman, 1980a; Dunbar et al., 1981; Sacco et al., 1981). Although only three glycoprotein families of the human ZP have been identified, each may be composed of more than one core protein. In the pig, for example, nonreduced ZP3 migrates as a diffuse band of $M_{\mathrm{r}}=\sim 55000$, but, upon deglycosylation, yields two protein bands of lower molecular weight (Hedrick \& Wardrip, 1986; Sacco et al., 1986).

The two-dimensional electrophoretic pattern of human ZP1 and ZP2 depends upon reduction with 2-mercaptoethanol. Under non-reducing conditions, ZP1 and ZP2 (ZP1/ZP2) comigrate to a range of 92000-120000/pI 4.4 4.5. After reduction, ZP2 becomes visible, migrating with an observed $M_{\mathrm{r}}$ that is $28000-42000$ less than the non-reduced molecule. After reduction, ZP1 also displays a lower observed range, 2000-10000 less than the non-reduced molecule. Reduced ZP1 and ZP2 may be subunits of a heterodimeric molecule (ZP1/ZP2), held together by inter-molecular disulphide bonds. However, the additive molecular weight of ZP1 and ZP2 seen after reduction represents a molecule much larger than that observed under non-reducing conditions for the ZP1/ZP2 complex. Therefore, we believe such a heterodimeric structure is unlikely.

The observed molecular weight range for reduced human ZP1 is almost entirely within the observed range for the non-reduced sample. Therefore, it is possible that ZP1 is not an oligomeric molecule, but instead may contain intramolecular disulphide bonds that cause the molecule to migrate to a different observed molecular weight when the sample is reduced. This anomalous behaviour is characteristic for glycoproteins, several of which migrate to lower observed molecular weights when reduced (Lambin, 1978). The large difference in observed molecular weights for nonreduced and reduced $\mathrm{ZP} 2$, however, is more consistent with an oligomeric structure. Although we have been unable specifically to identify radiolabelled subunits associated with ZP1 or ZP2, an oligomeric structure for either molecule cannot be completely ruled out. 
Our studies reveal that pools of zonae isolated from human eggs classified as fertilized display a dramatic decrease in the observed labelling of ZP1, and this modification is only seen if the sample is reduced. Modification of a mouse zona protein after fertilization has also been reported by Bleil et al. (1981). This modification involves a shift in the observed molecular weight from 120000 (mouse ZP2) to 90000 (mouse ZP2f), and as noted for human ZP1, is only seen after reduction. Bleil et al. (1981) conclude that mouse ZP2 is proteolytically cleaved by a cortical granule protease, but intramolecular disulphide bonds prevent the release of peptide fragments. Bleil et al. (1981) did not observe a large radiolabelled peptide fragment to account for the 30000 difference between ZP2 and $\mathrm{ZP} 2 \mathrm{f}$.

Although there is a dramatic decrease of human ZP1 from fertilized eggs, we have not yet determined where a modified ZP1 molecule resides. It is possible that ZP1 is proteolytically cleaved into fragments that are not iodinated or are iodinated but are too small to be recovered by our current methods. Although it is not apparent in the gel photographs, on original autoradiographs it appears, based upon relative band intensities, that a modified ZP1 molecule may co-migrate with ZP2 after reduction. This would involve the loss of $M_{\mathrm{r}}$ of about 35000 from ZP1 after fertilization and reduction, a fragment loss similar in size to that observed in the mouse.

We have also observed on autoradiographs of human ZP isolated from fertilized eggs under nonreducing conditions a radiolabelled zona component that migrates to a position where we typically see reduced $\mathrm{ZP} 2$ from non-fertilized eggs (arrow, Fig. 3a). We are unable to tell whether this component originates from ZPI or ZP2, and it could result from fertilization-associated modification of either molecule. Therefore, it is possible that both ZPI and ZP2 may be modified after fertilization. Resolution of this problem will require separation and purification of non-reduced ZP1 and ZP2 from unfertilized as well as fertilized eggs. This is complicated because ZP1 and ZP2 co-migrate unless reduced. The behaviour of these zona proteins, however, may be indicative of a closely related structure for the two components. Structural and chemical similarity of individual zona proteins has been demonstrated for pig zonae, for which $M_{\mathrm{r}} 25000$ and 65000 families have been shown not only to be compositionally similar to the $M_{\mathrm{r}} 90000$ family, but may also be derived from the $M_{\mathrm{r}} 90000$ family by hydrolytic processing (Wardrip \& Hedrick, 1985; Hedrick \& Wardrip, 1986). The $M_{\mathrm{r}} 65000$ and 25000 zona glycoproteins are apparently subunits of a heterodimer, which co-migrates with the $M_{\mathrm{r}} 90000$ family under non-reducing conditions. The individual $M_{\mathrm{r}}$ 65000 and 25000 families are revealed only after reduction with 2-mercaptoethanol, and without total loss of the $M_{\mathrm{r}} 90000$ family.

The modification of human ZP1 after fertilization is not always complete; there is variability in the observed decrease in labelling of ZP1. This characteristic is similar to that described for the mouse, for which ZP2 and ZP2f are observed after reduction (Bleil et al., 1981). Bleil et al. (1981) also report the occasional presence of ZP2f from unfertilized eggs. In the mouse, this is due to spontaneous activation of eggs associated with extended incubation periods (Aonuma et al., 1978; Gulyas, 1980). Spontaneous activation of human eggs has been reported to occur by some authors (Rosseau et al., 1977) but not others (Sathananthan \& Trounson, 1982). In our studies, we have not observed fertilization-associated zona modifications from pools of eggs classified as unfertilized. We conclude that the modification may not involve all ZPl molecules, thereby accounting for the observed variability. There may also be contamination of the pool with unfertilized eggs.

Presently, we can only presume that the modification of ZP1 after fertilization is responsible for a zona block to polyspermy. Other changes in the human zona pellucida may occur after fertilization but remain undetected by electrophoresis. This is also true for other species: although mouse ZP3 from fertilized eggs appears unaltered on polyacrylamide gels, it no longer possesses sperm receptor activity nor can it induce the acrosome reaction, both qualities which can be attributable to ZP3 isolated from unfertilized eggs (Bleil \& Wassarman, 1980b, 1983). Mouse ZP2, which is modified after fertilization, has been shown to bind to sites only on acrosome-reacted spermatozoa; these sites may become exposed after the acrosome reaction (Bleil \& Wassarman, 1986). 
We thank Dr Susan Fisher for critical reading of the manuscript; Dr Luther Talbert, director of the UNC-IVF-ET program, for continued support; and Dr Don P. Wolf for contributing zonae pellucidae to the project. Supported by NIH grant HD-14232 to M.O'R., and a postdoctoral fellowship to R.S. from Family Health International.

\section{References}

Anderson, N.L. \& Anderson, N.G. (1979) Microheterogeneity of serum transferrin, haptoglobulin and $\alpha_{2}$ HS-glycoprotein examined by high resolution twodimensional electrophoresis. Biochem. Biophys. Res. Commun. 88, 258-265.

Aonuma, S., Kabe, M., Kawai, Y. \& Kawaguchi, M. (1978) The change in solubility properties of zona pellucida to proteases related to fertilizability of mouse ova in vitro. Chem. Pharm. Bull. 26, 405-410.

Bleil, J.D. \& Wassarman, P.M. (1980a) Structure and function of the zona pellucida: identification and characterization of the proteins of the mouse oocyte's zona pellucida. Devl Biol. 76, 185-202.

Bleil, J.D. \& Wassarman, P.M. (1980b) Mammalian sperm-egg interaction: identification of a glycoprotein in mouse egg zonae pellucidae possessing receptor activity for sperm. Cell 20, 873-882.

Bleil, J.D. \& Wassarman, P.M. (1983) Sperm-egg interactions in the mouse: sequence of events and induction of the acrosome reaction by a zona pellucida glycoprotein. Devl Biol. 95, 317-324.

Bleil, J.D. \& Wassarman, P.M. (1986) Autoradiographic visualization of the mouse egg's sperm receptor bound to sperm. J. Cell Biol. 102, 1363-1371.

Bleil, J.D., Beall, C. \& Wassarman, P.M. (1981) Mammalian sperm-egg interaction: fertilization of mouse eggs triggers modification of the major zona pellucida glycoprotein, ZP2. Devl Biol. 86, 189-197.

Cherr, G., Lambert, H., Meizel, S. \& Katz, D. (1986) In vitro studies of the golden hamster sperm acrosome reaction: completion on the zona pellucida and induction by homologous soluble zonae pellucidae. Devl Biol. 114, 119-131.

Crozet, N. \& Dumont, M. (1984)The site of the acrosome reaction during in vivo penetration of the sheep oocyte. Gamete Res. 10, 97-105.

Dunbar, B. (1983) Morphological, biochemical and immunochemical characterization of the mammalian zona pellucida. In Mechanism and Control of Animal Fertilization, pp. 139-175. Ed. J. F. Hartmann. Academic Press, New York.

Dunbar, B.S. \& Wolgemuth, D. (1984) Structure and function of the mammalian zona pellucida, a unique extracellular matrix. Modern Cell Biol. 3, 77-111.

Dunbar, B.S., Liu, C. \& Sammons, D. W. (1981) Identification of the three major proteins of porcine and rabbit zonae pellucidae by high resolution twodimensional gel electrophoresis: comparison with serum, follicular fluid, and ovarian cell proteins. Biol. Reprod. 24, 1111-1124.

Dunbar, B.S., Wardrip, N.J. \& Hedrick, J.L. (1980) Isolation, physiochemical properties, and macromolecular composition of zona pellucida from porcine oocytes. Biochemistry, N.Y. 19, 356-365.

Florman, H. \& Storey, B. (1982) Mouse gamete inter- actions: the zona pellucida is the site of the acrosome reaction leading to fertilization in vitro. Devl Biol.91, 121-130.

Gulyas, B.J. (1980) Cortical granules of mammalian eggs. Int. Rev. Cytol. 63, 357-392.

Hartmann, J. (1983) Mammalian fertilization: gamete surface interactions in vitro. In Mechanism and Control of Animal Fertilization, pp. 325-359. Ed. J. F. Hartmann. Academic Press, New York.

Hedrick, J.L. \& Wardrip, N.J. (1986) Isolation of the zona pellucida and purification of its glycoprotein families from pig oocytes. Analyt. Biochem. 157, $63-70$.

Hedrick, J.L., Wardrip, N.J. \& Berger, T. (1987) Differences in the macromolecular composition of the zona pellucida isolated from pig oocytes, eggs, and zygotes. J. exp. Zool. 241, 257-262.

Hsu, C.-H. \& Kingsbury, D.W. (1982) Contribution of oligosaccharide sulfation to the charge heterogeneity of a viral glycoprotein. J. biol. Chem. 257, 9035-9038.

Lambin, P. (1978) Reliability of molecular weight determination of proteins by polyacrylamide gradient gel electrophoresis in the presence of sodium dodecyl sulfate. Analyt. Biochem. 85, 114-125.

O'Farrell, P.H. (1975) High resolution two-dimensional electrophoresis. J. biol. Chem. 250, 4007-4021.

O'Rand, M.G. \& Fisher, S.J. (1987) Localization of zona pellucida binding sites on rabbit spermatozoa and induction of the acrosome reaction by solubilized zonae. Devl Biol. 119, 551-559.

O'Rand, M.G., Welch, J.E. \& Fisher, S.J. (1986a) Sperm membrane and zona pellucida interactions during fertilization. In Molecular and Cellular Aspects of Reproduction, pp. 131-144. Eds D. S. Dhindsa \& O. P. Bahl. Plenum Publishing Corp., New York.

O'Rand, M.G., Herman, B., Diguiseppi, J., Halme, J., Hammond, M.G. \& Talbert, M. (1986b) Analysis of deoxyribonucleic acid distribution in noncleaving oocytes from patients undergoing in vitro fertilization. Fert. Steril. 46, 452-460.

Pearson, T.W. \& Anderson, N.L. (1983) Use of highresolution two-dimensional gel electrophoresis for analysis of monoclonal antibodies and their specific antigens. Methods Enzymol. 92, 196-220.

Rousseau, P., Meda, P., Lecart, C., Haumont, S. \& Ferin, J. (1977) Cortical granule release in human follicular oocytes. Biol. Reprod. 16, 104-111.

Sacco, A., Yurewicz, E., Subramanian, M. \& DeMayo, F. (1981) Zona pellucida composition: species cross reactivity and contraceptive potential of antiserum to a purified pig zona antigen (PPZA). Biol. Reprod. 25, 997-1008.

Sacco, A., Yurewicz, E. \& Subramanian, M. (1986) Carbohydrate influences the immunogenic and antigenic characteristics of the ZP3 macromolecule $\left(M_{\mathrm{r}}\right.$ 
55000 ) of the pig zona pellucida. J. Reprod. Fert. 76, Wardrip, N.J. \& Hedrick, J.L. (1985) Pig zona pellucida $575-586$.

Sathananthan, A.H. \& Trounson, A.O. (1982) Ultrastructural observation on cortical granules in human follicular oocytes cultured in vitro. Gamete Res. 5, 191-198.

Schmell, E., Gulyas, B. \& Hedrick, J. (1983) Egg surface changes during fertilization and the molecular mechanism of the block to polyspermy. In Mechanism and Control of Animal Fertilization, pp. 365-413. Ed. J. F. Hartman. Academic Press, New York. $25 \mathrm{~K}$ and $65 \mathrm{~K}$ glycoproteins are derived from hydrolysis and reduction of the $90 \mathrm{~K}$ family. $J$. Cell Biol. 101, 378a, abstr.

Yanagimachi, R. (1977) Specificity of sperm-egg interaction. In Immunobiology of Gametes, pp. 187-207. Eds M. Edidin \& M. H. Johnson. Cambridge University Press.

Received 7 April 1987 